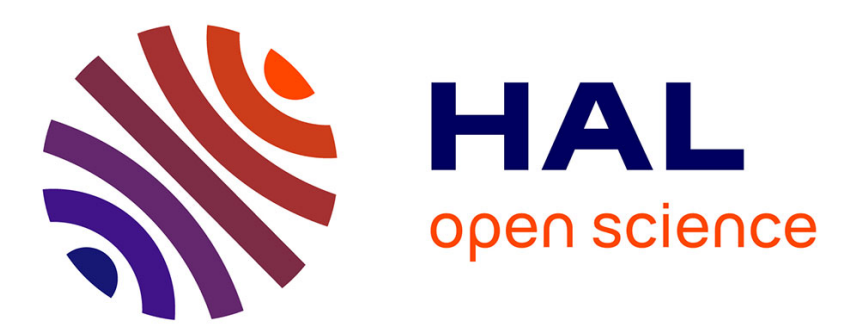

\title{
Conversion thermoélectrique par transitions de phase avec des matériaux ferroélectriques
}

\author{
R. Briot, P. Gonnard, Y. Fetiveau
}

\section{To cite this version:}

R. Briot, P. Gonnard, Y. Fetiveau. Conversion thermoélectrique par transitions de phase avec des matériaux ferroélectriques. Revue de Physique Appliquée, 1978, 13 (7), pp.366-371. 10.1051/rphysap:01978001307036600 . jpa-00244461

HAL Id: jpa-00244461

https://hal.science/jpa-00244461

Submitted on 1 Jan 1978

HAL is a multi-disciplinary open access archive for the deposit and dissemination of scientific research documents, whether they are published or not. The documents may come from teaching and research institutions in France or abroad, or from public or private research centers.
L'archive ouverte pluridisciplinaire HAL, est destinée au dépôt et à la diffusion de documents scientifiques de niveau recherche, publiés ou non, émanant des établissements d'enseignement et de recherche français ou étrangers, des laboratoires publics ou privés. 


\title{
CONVERSION THERMOÉLECTRIQUE PAR TRANSITIONS DE PHASE AVEC DES MATÉRIAUX FERROÉLECTRIQUES
}

\author{
R. BRIOT, P. GONNARD et Y. FETIVEAU \\ I.N.S.A. de Lyon, Département de Génie Electrique 504, \\ 20, avenue Albert-Einstein, 69621 Villeurbanne Cedex, France \\ (Reçu le 15 décembre 1977, révisé le 20 mars 1978, accepté le 10 avril 1978)
}

\begin{abstract}
Résumé. - La forte variation de la polarisation rémanente d'une céramique ferroélectrique lors d'un changement de phase consécutif à un échauffement ou à un refroidissement de l'échantillon, est à l'origine des convertisseurs thermoélectriques décrits dans cet article. Le principe de fonctionnement, les résultats expérimentaux obtenus et les rendements - expérimentaux et théoriques - sont étudiés à une transition ferroélectrique-antiferroélectrique, lors d'essais en descente de température ; la comparaison est faite avec les résultats obtenus au cours d'une transition ferroélectrique-antiferroélectrique ou ferroélectrique-paraélectrique en montée de température.
\end{abstract}

\begin{abstract}
Thermoelectric converters using ferroelectric ceramics are described in this paper. The large loss of the remanent polarization during a phase transition with rising or decreasing temperature is used in these converters. The principle of operation is reported ; experimental results, experimental and theoretical efficiencies are given for a ferroelectric-antiferroelectric phase transition during a drop in temperature. A comparative study with the results obtained during a ferroelectric-antiferroelectric or ferroelectric-paraelectric phase transition with rising temperature is achieved.
\end{abstract}

1. Introduction. - Un cristal ferroélectrique idéal monodomaine possède en l'absence de champ appliqué une polarisation spontanée. Un échantillon ferroélectrique fritté est constitué de domaines dont la polarisation spontanée est orientée au hasard. Le traitement de polarisation d'une céramique ferroélectrique consiste, après métallisation de deux armatures, à orienter par un champ électrique tous les domaines de polarisation spontanée dans une direction proche du champ appliqué et compatible avec la structure.

Après annulation du champ, il subsiste une polarisation rémanente c'est-à-dire des charges électriques liées à la céramique compensées par des charges libres sur les armatures : le champ électrique est donc nul aux bornes de l'échantillon.

On peut récupérer de l'énergie électrique en soumettant cet échantillon à une faible variation de température (ou de contrainte) tout en restant dans une phase ferroélectrique (F). La variation de la polarisation rémanente est alors réversible et les charges libres produisent un courant dans un circuit fermé sur la céramique : effet pyroélectrique (ou piézoélectrique).
Dans le cas d'une variation de température (ou de contrainte) de grande amplitude, on peut supprimer irréversiblement la polarisation rémanente soit par réorientation au hasard des domaines de polarisation, soit par induction d'une phase non polaire paraélectrique $(\mathrm{Pa})$ ou antiferroélectrique (AF).

Plusieurs types de convertisseurs thermoélectriques irréversibles ont été étudiés sur le plan théorique : conversion par variation de la polarisation spontanée [1] ou par transition forcée F-Pa [2].

Notons encore un autre processus de conversion d'énergie thermoélectrique dont le principe repose sur l'augmentation de l'énergie d'un condensateur lorsque sa capacité diminue, à charge électrique constante [3]. En effet la constante diélectrique de ces matériaux varie en fonction de la température et passe par un maximum aigu au point de Curie.

Ces différents convertisseurs n'ont pas fait en général l'objet d'études expérimentales très approfondies.

Les capteurs pyroélectriques réversibles sont utilisés pour la détection du rayonnement infrarouge (caméras infrarouge de télévision) $[4,5]$.

Le principe de fonctionnement du convertisseur 
thermoélectrique que nous avons étudié repose sur la variation de la polarisation rémanente d'un échantillon ferroélectrique lors d'un changement de phase $\mathrm{F} \rightarrow \mathrm{AF}$ en descente de température.

Après avoir exposé le principe du convertisseur thermoélectrique, nous analyserons les résultats expérimentaux obtenus avec des échantillons de zirconotitanate de plomb dopés (PZT) et nous donnerons un calcul du rendement de conversion. Nous ferons ensuite la comparaison avec les résultats obtenus avec d'autres matériaux PZT en montée de température (transition $\mathrm{F} \rightarrow \mathrm{AF} \rightarrow \mathrm{Pa}$ ou $\mathrm{F} \rightarrow \mathrm{Pa}$ ).

2. Conversion d'énergie thermoélectrique irréversible par changement de phase $\mathbf{F} \rightarrow \mathbf{A F}$ en descente de température. - 2.1 PRINCIPE DE FONCTIONNEMENT. - Les échantillons utilisés sont des céramiques ferroélectriques PZT dures (de composition proche de $\mathrm{PbZrO}_{3}$ ) dopées présentant en descente de température la succession de phases $\mathrm{Pa} \rightarrow \mathrm{F} \rightarrow \mathrm{AF}$. Lors du changement de phase $\mathrm{F} \rightarrow \mathrm{AF}$, la discontinuité de la polarisation rémanente $P_{\mathrm{r}}$ est plus prononcée que lors du changement de phase $\mathrm{F} \rightarrow \mathrm{Pa}$ (Fig. 1).

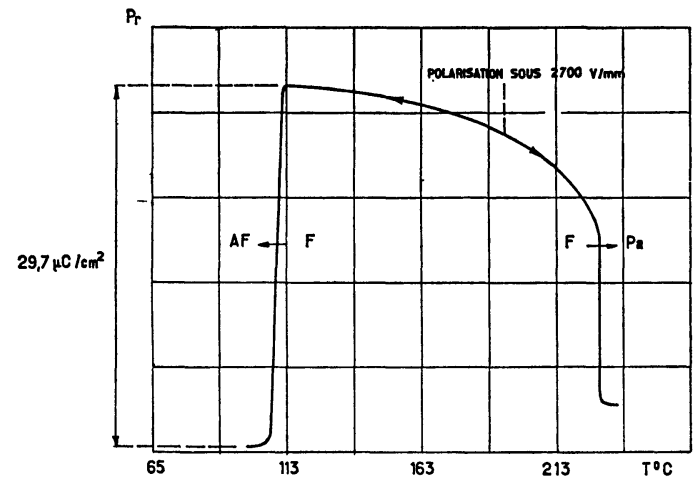

FIG. 1. - Charges électriques libérées en court-circuit en fonction de la température $\mathrm{PbZr}_{0,965} \mathrm{Ti}_{0,035} \mathrm{O}_{3}+0,01 \mathrm{CaO}$.

[Short-circuit charge versus temperature $\mathrm{PbZr}_{0.965} \mathrm{Ti}_{0.035} \mathrm{O}_{3}+0.01 \mathrm{CaO}$.]

Le diagramme énergétique du cycle de conversion non linéaire idéal est représenté par la figure $2, D$ étant l'induction, $E$ le champ électrique et $\varepsilon_{\mathrm{AF}}$ la constante diélectrique en phase $\mathrm{AF}$. Le champ $E_{\mathrm{F}}$ nécessaire pour induire une phase ferroélectrique, varie avec la température et décroît quand celle-ci augmente (courbe (a) Fig. 7).

Le principe de fonctionnement du convertisseur est le suivant :

Inverseur en position 1. - En $\mathrm{O}$, chauffage de l'échantillon en court-circuit jusqu'à la température $T_{2}$ de polarisation en phase ferroélectrique puis, à température constante, polarisation de l'échantillon sous un champ électrique $E_{1}$ supérieur au champ $E_{\mathrm{F}}$ (cycle Oabcd). En d, on retrouve la polarisation rémanente $\operatorname{Pr}$ après annulation du champ.
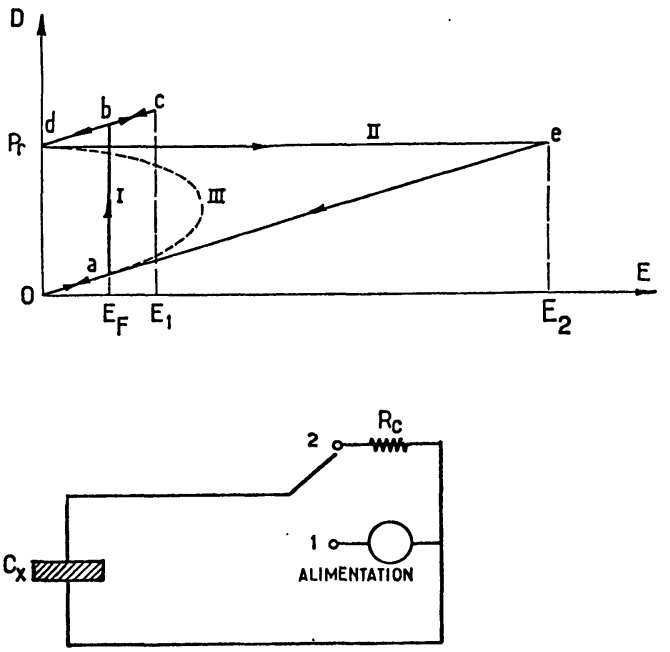

$R_{C}$ : Resistance de charge . $C_{X}$ : ceramique ferroelectrique

FIG. 2. - Diagramme énergétique du cycle de conversion théorique.

[Energetic diagram of the theoretical converter.]

Circuit ouvert. - d-e : refroidissement rapide de l'échantillon jusqu'à la température $T_{1}$ en circuit ouvert. Les charges électriques ne peuvent pas s'écouler à l'extérieur, on atteint le champ $E_{2}$ égal à $P_{\mathrm{r}} / \varepsilon_{\mathrm{AF}}$ aux bornes du condensateur ferroélectrique.

Inverseur en position $2 .-\mathrm{e}-0$ : on branche la résistance de charge $R_{\mathrm{c}}$ et la totalité des charges de polarisation est récupérée dans le circuit extérieur.

La courbe I correspond à la courbe de première polarisation du cycle d'hystérésis. La courbe II correspond au cycle de conversion théorique idéal. La valeur de $\varepsilon_{\mathrm{AF}}$ étant faible dans les PZT utilisés, le champ $E_{2}=P_{\mathrm{r}} / \varepsilon_{\mathrm{AF}}$ est environ cent fois plus élevé que le champ $E_{\mathrm{F}}$.

Expérimentalement, la résistance de charge $R_{\mathrm{c}}$ est branchée dès le début du refroidissement de l'échantillon (en d) ; la courbe expérimentale est alors schématisée par le tracé III.

$\mathrm{Au}$ cours de la dépolarisation, l'augmentation du champ électrique aux bornes de $R_{\mathrm{c}}$ étend la transition $\mathrm{F} \rightarrow \mathrm{AF}$ sur un plus grand intervalle de température et la repousse vers les basses températures, ce qui se traduit par un accroissement du domaine ferroélectrique (courbe (b) Fig. 7) [7].

Le bilan énergétique du cycle de conversion théorique est résumé par le tableau I. $\rho_{0}$ est la masse volumique du matériau en $\mathrm{g} / \mathrm{cm}^{3}, C_{p}$ sa chaleur spécifique à champ constant en $\mathrm{J} / \mathrm{g} .{ }^{\circ} \mathrm{C}, \Delta Q_{\mathrm{AF} \rightarrow \mathrm{F}} \mathrm{sa}$ chaleur de transition lors du changement de phase $\mathrm{AF} \rightarrow \mathrm{F}$ en $\mathrm{J} / \mathrm{g}$.

L'énergie électrique fournie à l'échantillon pour le polariser correspond à l'aire Oabd alors que l'énergie électrique récupérée correspond à l'aire Ode. Il y a donc un gain en énergie électrique. 
TABleau I

Energie électrique

En 0, chauffage en court-circuit

Polarisation à température constante $T_{2}$

Refroidissement en circuit ouvert

$R_{\mathrm{c}}$ branchée sur la céramique

Bilan énergétique

$$
\begin{gathered}
\overline{0} \\
-P_{\mathrm{r}} E_{\mathrm{F}} \\
0 \\
P_{\mathrm{r}}^{2} / 2 \varepsilon_{\mathrm{AF}} \\
\frac{P_{\mathrm{r}}^{2}}{2 \varepsilon_{\mathrm{AF}}}-P_{\mathrm{r}} E_{\mathrm{F}}
\end{gathered}
$$

Energie thermique

$$
\begin{gathered}
\rho_{0} C_{p}\left(T_{2}-T_{1}\right)+\rho_{0}\left|\Delta Q_{\mathrm{AF} \rightarrow \mathrm{F}}\right| \\
0 \\
\text { non récupérée } \\
0
\end{gathered}
$$$$
\rho_{0} C_{p}\left(T_{2}-T_{1}\right)+\rho_{0}\left|\Delta Q_{\mathrm{AF} \rightarrow \mathrm{F}}\right|
$$

Nous définirons le rendement $\eta$ de la conversion de la manière suivante :

$$
\eta=\frac{W_{\mathrm{ER}}-W_{\mathrm{EF}}}{W_{\mathrm{T}}}
$$

avec

$W_{\mathrm{ER}}$ : énergie électrique récupérable (rendement théorique) ou récupérée (rendement expérimental),

$W_{\mathrm{EF}}$ : énergie électrique fournie pour polariser l'échantillon,

$W_{\mathrm{T}}$ : énergie thermique nécessaire pour chauffer l'échantillon.

On obtient donc un rendement théorique :

$$
\eta_{\mathrm{th}}=\frac{P_{\mathrm{r}}^{2} / 2 \varepsilon_{\mathrm{AF}}-P_{\mathrm{r}} E_{\mathrm{F}}}{\rho_{0} C_{p}\left(T_{2}-T_{1}\right)+\rho_{0}\left|\Delta Q_{\mathrm{AF} \rightarrow \mathrm{F}}\right|} .
$$

2.2 Matériaux. - Le choix des matériaux est lié à la nature de la transition $\mathrm{F} \rightarrow \mathrm{AF}$ c'est-à-dire à l'existence d'une hystérésis en température entre la montée et la descente de température, et à la grande mobilité de cette transition sous champ électrique [8].

Nous avons étudié tout particulièrement un échantillon de composition $\mathrm{PbZr}_{0,965} \mathrm{Ti}_{0,035} \mathrm{O}_{3}+1 \%$ en mole de $\mathrm{CaO}$ que nous noterons PZT 96,5/3,51\% $\mathrm{CaO}$ dont les températures de transition $\mathrm{F} \leftrightarrows \mathrm{AF}$ en montée et en descente de température sont à champ nul respectivement de $180^{\circ} \mathrm{C}$ et $113^{\circ} \mathrm{C}$.

Les céramiques sont fabriquées au laboratoire suivant la méthode classique des mélanges d'oxydes. Les échantillons sont métallisés puis collés sur un support métallique qui sert de radiateur thermique. L'ensemble est enrobé à l'aide d'un isolant électrique adéquat afin d'éviter les claquages électriques extérieurs à la céramique. Le chauffage de l'échantillon se fait par l'intermédiaire d'un four [6].

2.3 CyCle EXPÉRIMENTAL. - Le cycle de conversion expérimental est le suivant :

- chauffage de la céramique en phase ferroélectrique (vers $180^{\circ} \mathrm{C}$ ),

- polarisation de l'échantillon (environ $3000 \mathrm{~V} / \mathrm{mm}$ ),

- dépolarisation de l'échantillon sur une résistance de charge $R_{\mathrm{c}}$ au cours d'un refroidissement lent (libre) ou rapide (avec ventilation). La valeur de $R_{\mathrm{c}}$ est comprise entre $1000 \mathrm{M} \Omega$ et $120000 \mathrm{M} \Omega$ pour des échantillons de $0,68 \mathrm{~cm}^{2}$ de surface environ.

La courbe $V=f(t)$ de la tension aux bornes de $R_{\mathrm{c}}$ enregistrée en fonction du temps donne la variation du champ électrique aux bornes de la céramique. Si $e$ représente l'épaisseur de l'échantillon et $S$ sa surface, nous avons $E=V / e=f(t)$ en $\mathrm{V} / \mathrm{mm}$.

Posons $R_{\mathrm{c}}=\rho \frac{e}{S}$ avec $\rho$ résistivité équivalente de la résistance de charge.

Dans ce cas $\frac{\Delta Q}{S}=\int \frac{E}{\rho} \mathrm{d} t$ représente la densité de charges électriques récupérées en $\mu \mathrm{C} / \mathrm{cm}^{2}$ et

$$
\frac{W}{e . S}=\int \frac{E^{2}}{\rho} \mathrm{d} t
$$

l'énergie électrique récupérée par unité de volume en $\mathrm{J} / \mathrm{cm}^{3}$.

2.4 Résultats expérimentauX. - Pour chaque épaisseur de l'échantillon, nous avons enregistré les courbes $E=f(t)$ pour différentes résistances de charge et pour deux vitesses de refroidissement (Fig. 3).

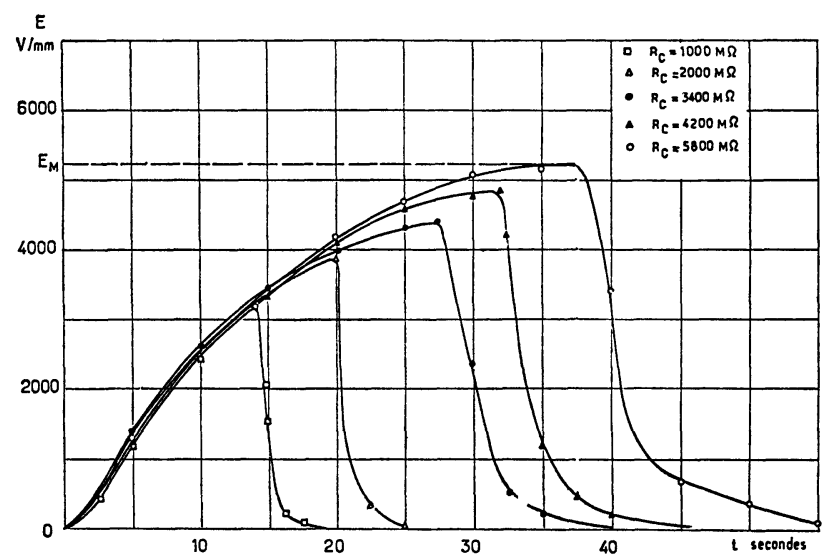

FIG. 3. - Champ électrique en fonction du temps. Refroidissement rapide. PZT 96,5/3,5 $1 \% \mathrm{CaO} . e=0,68 \mathrm{~mm} ; S=0,68 \mathrm{~cm}^{2}$.

[Electric field versus time-Speedy cooling. PZT 96.5/3.5 1\% CaO. $e=0.68 \mathrm{~mm} ; S=0.68 \mathrm{~cm}^{2}$.]

La tension augmente aux bornes de $R_{\mathrm{c}}$ tant que la céramique fonctionne en générateur de charges; le 
champ atteint une valeur maximale $E_{\mathrm{M}}$ et on assiste ensuite à la décharge du condensateur ferroélectrique.

$\mathrm{Au}$ cours de tous les essais effectués, la densité de charges récupérées est sensiblement constante et égale à $28 \mu \mathrm{C} / \mathrm{cm}^{2}$.

Les figures 4 et 5 représentent les variations du champ électrique $E_{\mathrm{M}}$ et de l'énergie électrique récupérée en fonction de $\rho$.

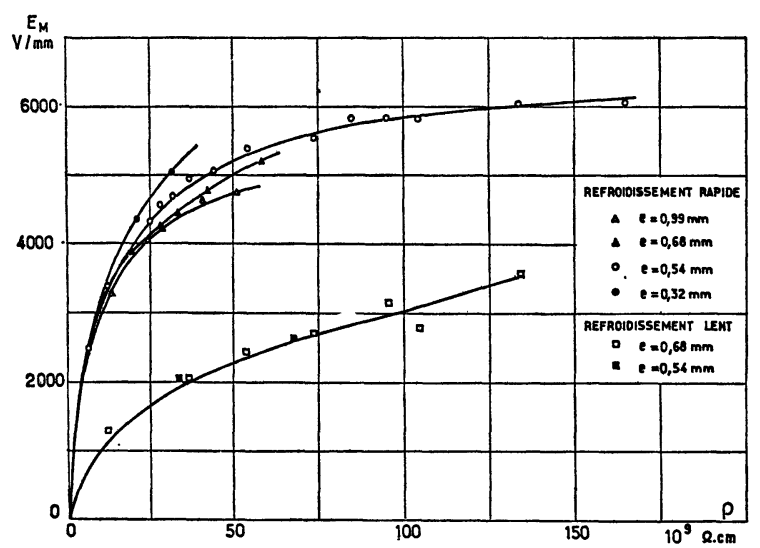

Fig. 4. - Champ maximum $E_{\mathrm{M}}$ en fonction de la résistivité équivalente $\rho$. PZT $96,5 / 3,51 \% \mathrm{CaO} ; S=0,68 \mathrm{~cm}^{2}$.

[Maximum electric field $E_{\mathrm{M}}$ versus equivalent specific resistivity $\rho$. PZT $96.5 / 3.51 \% \mathrm{CaO} ; S=0.68 \mathrm{~cm}^{2}$.]

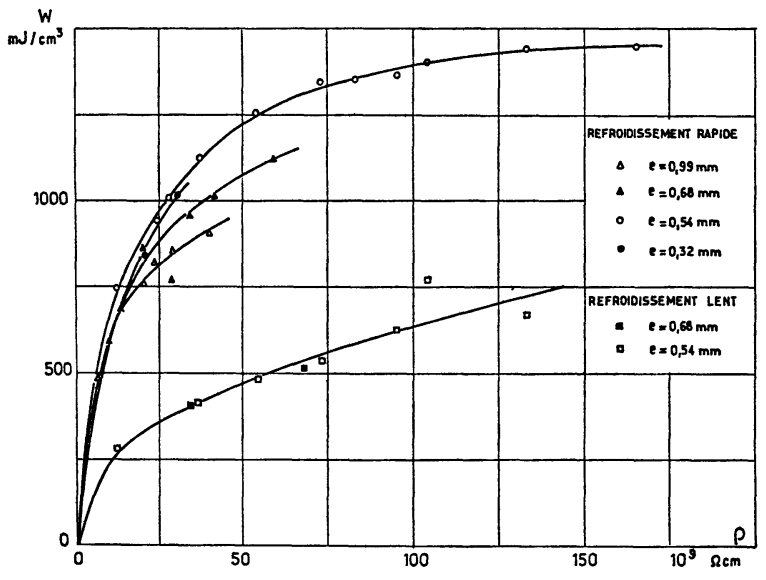

Fig. 5. - Energie récupérée $W$ en fonction de la résistivité équivalente $\rho$. PZT 96,5/3,51\% $\mathrm{CaO} ; S=0,68 \mathrm{~cm}^{2}$.

[Experimental collected energy versus equivalent specific resistivity $\rho$. PZT $96.5 / 3.51 \% \mathrm{CaO} ; S=0.68 \mathrm{~cm}^{2}$.]

L'énergie maximale a été libérée en refroidissement rapide par un échantillon d'épaisseur $0,54 \mathrm{~mm}$. En refroidissement lent, les résultats sont comparables quelle que soit l'épaisseur (tableau II).

\section{TABLEAU II}

\begin{tabular}{|c|c|c|c|}
\hline Refroidissement & $R_{\mathrm{c}}(\mathrm{M} \Omega)$ & $W\left(\mathrm{~J} / \mathrm{cm}^{3}\right)$ & $E_{\mathrm{M}}(\mathrm{V} / \mathrm{mm})$ \\
\hline ent $\quad$ - & $\overline{8200}$ & $\overline{0,76}$ & $\overline{2780}$ \\
\hline pide & 10600 & 1,44 & 6030 \\
\hline
\end{tabular}

Ces résultats correspondent à l'énergie maximale récupérée au cours des essais et non à l'énergie maximale récupérable expérimentalement. En effet, pour un échantillon de bonne qualité présentant une très grande résistance interne, nous ne trouvons pas dans les limites possibles de l'expérience (claquage extérieur à la céramique malgré l'enrobage effectué) d'adaptation d'énergie en fonction de la résistance de charge [9].

L'étude faite en fonction de la vitesse de refroidissement ne permet pas de tirer des conclusions générales sur les résultats obtenus. Au cours d'un refroidissement lent, le claquage extérieur à la céramique se produit pour un champ électrique beaucoup plus faible (environ $2800 \mathrm{~V} / \mathrm{mm}$ ) qu'au cours d'un refroidissement rapide (environ $6000 \mathrm{~V} / \mathrm{mm}$ ), mais dans ce cas le champ n'apparaît entre les électrodes que pendant un court instant.

Pour un champ $E_{\mathrm{M}}$ donné, l'énergie récupérée est sensiblement constante quel que soit le mode de refroidissement utilisé.

En refroidissement lent les résistances de charge utilisées sont grandes; il semble donc préférable d'utiliser un refroidissement rapide.

Les résultats obtenus avec un échantillon PZT $96,5 / 3,5 \quad 0,2 \% \quad \mathrm{Nd}_{2} \mathrm{O}_{3}$ sont comparables aux précédents. Pour une céramique de $0,5 \mathrm{~mm}$ d'épaisseur et de $0,69 \mathrm{~cm}^{2}$ nous avons obtenu sur une résistance de charge de $8200 \mathrm{M} \Omega$ en refroidissement rapide, une énergie de $1,14 \mathrm{~J} / \mathrm{cm}^{3}$ pour un champ électrique maximum de $5200 \mathrm{~V} / \mathrm{mm}$.

2.5 RENDEMENT DE CONVERSION. - Nous avons défini le rendement de conversion de la manière suivante :

$$
\eta=\left(W_{\mathrm{ER}}-W_{\mathrm{EF}}\right) / W_{\mathrm{T}} .
$$

La chaleur de transition $\Delta Q_{\mathrm{AF} \rightarrow \mathrm{F}}$ lors du changement de phase $\mathrm{AF} \rightarrow \mathrm{F}$ (environ $0,04 \mathrm{~J} / \mathrm{g}$ [8]) est négligeable. Nous prendrons pour $C_{p}$ une valeur moyenne donnée par la littérature soit $C_{p}=0,36 \mathrm{~J} / \mathrm{g} .{ }^{\circ} \mathrm{C}$.

Les énergies $W_{\mathrm{EF}}$ et $W_{\mathrm{ER}}$ théoriques sont définies à partir du diagramme de la figure 2. L'expression de l'énergie théorique récupérable $W_{\mathrm{ER}}$ n'est valable que si l'on considère une décharge sur une grande résistance et une valeur de $\varepsilon_{\mathrm{AF}}$ constante et indépendante de la température et du champ électrique.

Application numérique. - Echantillon

PZT 96,5/3,5 $1 \% \mathrm{CaO}$.

$P_{\mathrm{r}}=28 \mu \mathrm{C} / \mathrm{cm}^{2}$;

$\varepsilon_{\mathrm{AF}}=1,67 \times 10^{-9} \mathrm{~F} / \mathrm{m}$ (température ambiante); $\rho_{0}=7,7 \mathrm{~g} / \mathrm{cm}^{3} ;$

$T_{1}=25^{\circ} \mathrm{C} ; \quad T_{2}=174^{\circ} \mathrm{C} ;$

$$
E_{\mathrm{F}}=850 \mathrm{~V} / \mathrm{mm}
$$

déterminé à partir des cycles d'hystérésis . 
On obtient

$$
\begin{aligned}
& W_{\mathrm{EF}}=0,238 \mathrm{~J} / \mathrm{cm}^{3} \\
& W_{\mathrm{ER}}=23,5 \mathrm{~J} / \mathrm{cm}^{3}, \\
& W_{\mathrm{T}}=413 \mathrm{~J} / \mathrm{cm}^{3}
\end{aligned}
$$

soit un rendement théorique $\eta_{\mathrm{th}}=5,6 \%$.

Remarquons que le champ $P_{\mathrm{r}} / \varepsilon_{\mathrm{AF}}$ est nettement supérieur au champ de claquage du matériau; cette valeur du rendement semble donc utopique.

Par intégration graphique sur un diagramme énergétique expérimental (Fig. 6), nous obtenons $W_{\mathrm{EF}}=0,35 \mathrm{~J} / \mathrm{cm}^{3}$ et $W_{\mathrm{ER}}=1,44 \mathrm{~J} / \mathrm{cm}^{3}$. L'énergie thermique $W_{\mathrm{T}}$ est égale à la précédente. Le rendement expérimental $\eta_{\text {exp }}$ est dans ces conditions de $0,26 \%$.

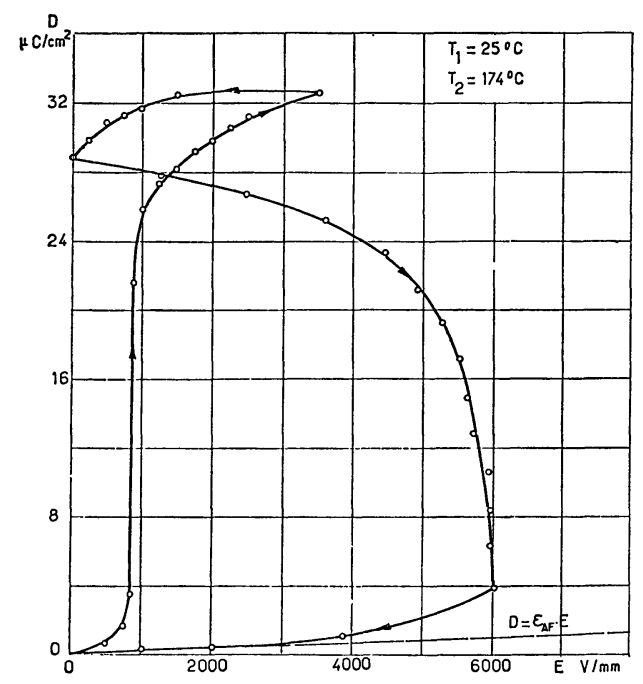

FIG. 6. - Diagramme énergétique du cycle de conversion expérimental. PZT 96,5/3,5 $1 \%$ CaO. $e=0,54 \mathrm{~mm} ; S=0,68 \mathrm{~cm}^{2}$. Refroidissement rapide $-R_{\mathrm{c}}=10600 \mathrm{M} \Omega$.

[Energetic diagram of the experimental converter. PZT 96.5/3.5 1\% CaO. $e=0.54 \mathrm{~mm} ; S=0.68 \mathrm{~cm}^{2}$. Speedy cooling

$$
-R_{\mathrm{c}}=10600 \mathrm{M} \Omega \text {.] }
$$

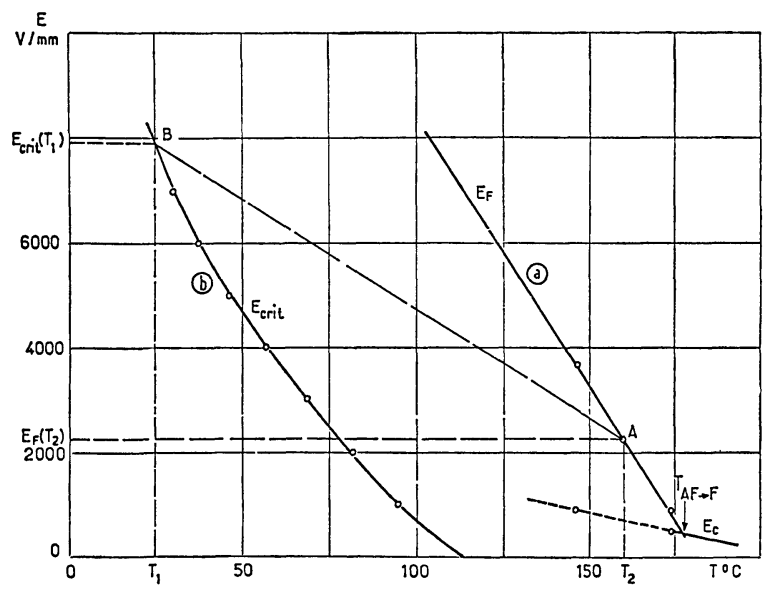

FIG. 7. - Diagramme champ électrique-température de transition. PZ1 96,5/3,5 $1 \% \mathrm{CaO}$.

[Electric field as function of transition temperature. PZT 96.5/3.5 $1 \% \mathrm{CaO}$.]
Sur la figure 7, nous avons porté dans le plan $E(T)$, la courbe $E_{\mathrm{F}}(T)$ (courbe a) obtenue à partir des cycles d'hystérésis et la courbe $E_{\text {crit }}(T)$ (courbe b) qui correspond au déplacement de la transition $\mathrm{F} \rightarrow \mathrm{AF}$ sous champ électrique en descente de température. Cette courbe représente le champ maximum que l'on pourra atteindre au cours du cycle de conversion.

Le champ $E_{\text {crit }}$ est obtenu en effectuant le cycle de conversion lorsque la céramique est branchée sur une résistance de charge de très grande valeur $(120000 \mathrm{M} \Omega)$. On peut assimiler un tel fonctionnement à un circuit ouvert dans les limites de l'expérience (champ électrique atteint de l'ordre de $6000 \mathrm{~V} / \mathrm{mm}$ ), l'écoulement des charges électriques étant très faible, environ $1 \mu \mathrm{C} / \mathrm{cm}^{2}$ en refroidissement rapide.

Soit $E_{\text {crit }}\left(T_{1}\right)$ le champ maximum que l'on peut atteindre à la température $T_{1}$ au cours du cycle de conversion (point B) et soit $\mathrm{A}$, le point d'abscisse $T_{2}$ et d'ordonnée $E_{\mathrm{F}}\left(T_{2}\right)$. L'énergie électrique maximale récupérée est sensiblement $P_{\mathrm{r}} E_{\text {crit }}$; le rendement théorique correspond alors au produit de la pente du segment $\mathrm{AB}$ par $P_{\mathrm{r}} / \rho_{0} C_{p}$.

Ce rendement sera d'autant plus grand que la pente de $\mathrm{AB}$ sera grande donc q $E_{\text {crit }}\left(T_{1}\right)$ sera grand ; le segment $\mathrm{AB}$ tend alors à devenir confondu avec la courbe $E_{\mathrm{F}}(T)$.

Le rendement limite correspond donc au produit de la pente de la courbe $E_{\mathrm{F}}(T)$ par $P_{\mathrm{r}} / \rho_{0} C_{p}$ soit avec ce matériau environ à $1 \%$.

3. Conversion thermoélectrique en montée de température. - Nous avons effectué quelques essais de conversion d'énergie en montée de température sur différents échantillons fabriqués au laboratoire.

(a) $\mathrm{Pb}_{0,99} \mathrm{Nb}_{0,02}\left|\left(\mathrm{Zr}_{0,73} \mathrm{Sn}_{0,27}\right)_{0,93} \mathrm{Ti}_{0,07}\right|_{0,98} \mathrm{O}_{3}$ (composition d'après Berlincourt [10])

$$
\mathrm{F}_{\mathrm{A}} \stackrel{129^{\circ} \mathrm{C}}{\longrightarrow} \mathrm{AF}_{\mathrm{B}} \stackrel{158^{\circ} \mathrm{C}}{\longrightarrow} \mathrm{Pa}
$$

(b) $\mathrm{PbHf}_{0,96} \mathrm{Ti}_{0,04} \mathrm{O}_{3}+1 \% \mathrm{Nb}_{2} \mathrm{O}_{5}$

$$
\mathrm{F}_{\mathrm{A}} \stackrel{55^{\circ} \mathrm{C}}{\longrightarrow} \mathrm{AF}_{\mathrm{B}} \stackrel{181^{\circ} \mathrm{C}}{\longrightarrow} \mathrm{Pa}
$$

(polarisation des céramiques à température ambiante sous $4000 \mathrm{~V} / \mathrm{mm}$ ).

(c) $\mathrm{PbZr}_{0,965} \mathrm{Ti}_{0,035} \mathrm{O}_{3}+1 \% \mathrm{Nb}_{2} \mathrm{O}_{5}$

$$
\mathrm{FA} \stackrel{41^{\circ} \mathrm{C}}{\longrightarrow} \mathrm{F}_{\mathrm{B}} \stackrel{221^{\circ} \mathrm{C}}{\longrightarrow} \mathrm{Pa} .
$$

L'énergie électrique récupérée et le champ maximal atteint en montée de température sont reportés dans le tableau III.

Ce mode de conversion ne permet pas de récupérer une énergie électrique supérieure à l'énergie de polarisation sauf dans le cas de l'échantillon (a) qui possède une température de transition $\mathrm{F} \rightarrow \mathrm{Pa}$ relativement basse. 
TABlEAU III

\begin{tabular}{|c|c|c|c|c|c|}
\hline $\begin{array}{l}\text { Mode de } \\
\text { conversion }\end{array}$ & Echantillon & $\begin{array}{l}\text { Energie maximale } \\
\text { récupérée } \\
\text { en } \mathrm{J} / \mathrm{cm}^{3}\end{array}$ & $\begin{array}{l}\text { Champ élect. } \\
\text { à l'adaptation } \\
\mathrm{V} / \mathrm{mm}\end{array}$ & $\begin{array}{l}\text { Température de } \\
\text { polarisation }\end{array}$ & $\begin{array}{l}\text { Energie de } \\
\text { polarisation } \\
\text { en } \mathrm{J} / \mathrm{cm}^{3}\end{array}$ \\
\hline - & - & - & - & - & - \\
\hline \multicolumn{6}{|c|}{ 1) Conversion thermoélectrique en descente de température } \\
\hline $\begin{array}{l}\mathrm{F} \rightarrow \mathrm{AF} \\
\mathrm{F} \rightarrow \mathrm{AF}\end{array}$ & $\begin{array}{l}\text { PZT 96,5/3,5 } 1 \% \mathrm{CaO} \\
\text { PZT 96,5/3,5 0,2\% } \mathrm{Nd}_{2} \mathrm{O}_{3}\end{array}$ & $\begin{array}{l}1,5 \\
1,14\end{array}$ & $\begin{array}{l}* 6000 \\
* 5200\end{array}$ & $\begin{array}{l}174^{\circ} \mathrm{C} \\
170^{\circ} \mathrm{C}\end{array}$ & $\begin{array}{l}0,24 \\
0,25\end{array}$ \\
\hline \multicolumn{6}{|c|}{$\begin{array}{l}\text { * Ce champ correspond au champ maximum atteint sans claquage } \\
\text { 2) Conversion thermoélectrique en montée de température }\end{array}$} \\
\hline $\begin{array}{l}\mathrm{F} \rightarrow(\mathrm{AF}) \rightarrow \mathrm{Pa} \\
\mathrm{F} \rightarrow \mathrm{AF} \\
\mathrm{F} \rightarrow(\mathrm{AF}) \rightarrow \mathrm{Pa} \\
\mathrm{F}_{\mathrm{A}} \rightarrow \mathrm{F}_{\mathrm{B}} \rightarrow \mathrm{Pa}\end{array}$ & $\begin{array}{c}\mathrm{Pb}_{0,99} \mathrm{Nb}_{0,02}\left[\left(\mathrm{Zr}_{0,73} \mathrm{Sn}_{0,27}\right)_{0,93} \mathrm{Ti}_{0,07}\right]_{0,98} \mathrm{O}_{3} \\
\text { PHT } 96 / 41 \sim \mathrm{Nb}_{2} \mathrm{O}_{5} \\
\text { PHT } 96 / 41 \% \mathrm{Nb}_{2} \mathrm{O}_{5} \\
\text { PZT } 96,5 / 3,51 \% \mathrm{Nb}_{2} \mathrm{O}_{5}\end{array}$ & $\begin{array}{l}0,34 \\
0,14 \\
0,26 \\
0,18\end{array}$ & $\begin{array}{l}3300 \\
1300 \\
3340 \\
1750\end{array}$ & $\begin{array}{l}\text { ambiante } \\
\text { ambiante } \\
\text { ambiante } \\
\text { ambiante }\end{array}$ & $\begin{array}{l}0,16 \\
0,78 \\
0,78 \\
0,40\end{array}$ \\
\hline
\end{tabular}

4. Conclusion. - En raison des pertes diélectriques importantes à haute température, la conversion d'énergie thermoélectrique en montée de température ne semble intéressante que pour des matériaux ayant un point de Curie peu élevé et possédant une polarisation rémanente la plus grande possible. Il convient de mentionner que le faible déplacement de la transition $\mathrm{F} \rightarrow \mathrm{Pa}$ avec le champ $[7,10]$ permet de réduire l'écart entre les températures $T_{1}$ et $T_{2}$. Au contraire, lors de l'existence d'une phase AF haute température précédée d'une phase $\mathrm{F}$, l'hystérésis importante de la transition avec le champ oblige à accroître l'écart entre $T_{1}$ et $T_{2}$ ce qui diminue le rendement.

Pour toutes ces raisons et compte tenu des résultats obtenus, il semble préférable d'effectuer la conversion en descente de température. Il n'en demeure pas moins que le rendement expérimental est inférieur à $1 \%$ en raison de l'énergie thermique nécessaire à l'élévation de température jusqu'à $T_{2}$.

Des applications ne semblent donc envisageables que si le coût de ce chauffage est faible ou nul (énergie solaire par exemple). Un accroissement du rendement ne pourrait être dû qu'à une diminution de la densité, de la chaleur spécifique des matériaux, de l'hystérésis en température, ou bien encore à une augmentation de la polarisation rémanente ou du rapport $\mathrm{d} E / \mathrm{d} T$ de la transition.

\section{Bibliographie}

[1] Fatuzzo, E., Kiess, H., NitsChe, R., J. Appl. Phys. 37 (1966) 510.

[2] Childress, J. D., J. Appl. Phys. 33 (1962) 1793.

[3] Clingman, W. H., Moore, R. G., J. Appl. Phys. 32 (1961) 675.

[4] Putley, E. H., Opt. Laser Technol. 3 (1971) 150.

[5] Felix, P., Réunion Française de Ferroélectricité, Nantes (septembre 1974).
[6] BRIot, R., Thèse Lyon 1977.

[7] Benguigui, L., Rev. Gén. Electr. 77 (1968) 938.

[8] Troccaz, M., Thèse Lyon 1975.

[9] Briot, R., Gonnard, P., Troccaz, M., Grange, G., Fetiveau, Y., C. R. Hebd. Séan. Acad. Sci. Paris 278 (1974) 973.

[10] BerlincourT, D., IEEE Trans. Sonics Ultrason. 15, $\mathrm{n}^{\circ} 2$ (1968) 89. 